\title{
Editorial: Artificial Intelligence for Mobile Robotic Networks
}

\author{
Huimin $\mathrm{Lu}^{1} \cdot \mathrm{Li} \mathrm{He}^{2} \cdot$ Quan $\mathrm{Zhou}^{3} \cdot{\text { Zongyuan } \mathrm{Ge}^{4}}^{4}$
}

Published online: 5 October 2017

(C) Springer Science+Business Media, LLC 2017

Editorial:

The integration of artificial intelligence and multimedia communication networks has become a topic of increasing interest for both researchers and developers from academia and industry worldwide. It is foreseeable that artificial intelligence will be the main approach to the next generation of mobile robotic networks. The explosive number of artificial intelligence methods and the increasing computational power of network-based computers have significantly extended the number of potential applications for mobile robotics. It has also brought new challenges to the artificial intelligence community. The aim of this special issue is to provide a platform to share up-todate scientific achievements in this field.

This special issue features six selected papers with high quality. The first article, "MT-AAAU: Design of Monitoring and Tracking for Anti-Abuse of Amateur UAV", proposed a monitoring UAV (M-UAV) for security purposes. In particular, the relevant design issues of M-UAV monitoring are discussed; possible solutions are given, and finally, the feasible communication architectures for coordinating multiple monitoring UAVs (MM-UAVs) for Anti-Abuse of Amateur UAV (AAAU) in no-fly zone are summarized.

Huimin Lu

dr.huimin.lu@ieee.org

Kyushu Institute of Technology, Kyushu, Japan

2 Qualcomm, San Diego, CA, USA

3 Nanjing University of Posts and Telecommunications, Nanjing, Jiangsu Province, China

4 IBM, Sydney, Australia
The second article titled "Anxiety Level Detection Using BCI of Miner's Smart Helmet" presented an emotional state evocation paradigm to find the brain area where the emotion feature is best. Then a fusion algorithm of the anxiety level is proposed to evaluate the miner's mental state by using the $\theta, \alpha$, and beta rhythms of EEG. Finally, the miner's smart helmet system is built to collect the human state which includes the mental parameters of the anxiety level, the fatigue level, the concentration level, and the environmental parameter in coal mine.

In the next article with the title "A Voting Aggregation Algorithm for Optimal Social Satisfaction", the authors investigated the applications of social choice and innovatively proposed a formula called Social Satisfaction Degree (SSD) for single-winner and multi-winner voting system.

Optical imaging is widely regarded as one of the most promising technologies for underwater observing networks. The fourth article entitled "Non-uniform Descattering and De-blurring of Underwater Images" proposed a hierarchical transmission fusion method and a color-line ambient light estimation method for high turbidity inhomogeneous underwater image restoration from a single input image. The proposed scheme overcomes the non-uniform scattering and blurring issues of the captured underwater images in underwater observing networks.

The fifth article, "Development of Mobile Magnetic Measurement System Using Laser Beam and Image Processing" proposed a method for new magnetic measurement using posture information. With this new method, it is possible to move magnetic sensor and to enable the magnetic measurement of the object.

The last article titled "Brain Intelligence: Go Beyond Artificial Intelligence" investigated a comprehensive review of deep learning algorithms and brain intelligence and how they can be applied in real world engineering. The proposed "Brain Intelligence (BI)" model generates 
new ideas about events without having experienced them by using artificial life with an imagine function. The authors conducted demonstrations of the developed BI intelligence learning model on automatic driving, precision medical care, and industrial robots.

Acknowledgements The guest editors are thankful to our reviewers for their effort in reviewing the manuscripts. We also thank the Edit-in-Chief, Dr. Imrich Chlamtac for his supportive guidance during the entire process. The special issue is sponsored by Leading Initiative for Excellent Young Research Program of Ministry of Education, Culture, Sports, Science and Technology of Japan (16809746), Grant in Aid for Scientific Research of JSPS (17 K14694), National Natural Science Foundation of China (61602089), Research Fund of The Telecommunications Advancement Foundation, Fundamental Research Developing Association for Shipbuilding and Offshore, Strengthening Research Support Project of Kyushu Institute of Technology, Kitakyushu Convention \& Visitors Association and Support Center for Advanced Telecommunications Technology Research Foundation.

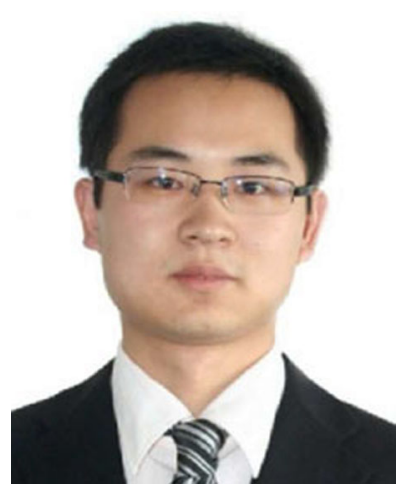

Huimin Lu received M.S. degrees in Electrical Engineering from Kyushu Institute of Technology and Yangzhou University in 2011. He received a Ph.D. degree in Electrical Engineering from Kyushu Institute of Technology in 2014. From 2013 to 2016, he was a JSPS research fellow at Kyushu Institute of Technology, Japan. Currently, he is an Assistant Professor in Kyushu Institute of Technology and an Excellent Young Researcher of MEXTJapan. His research interests include computer vision, robotics, artificial intelligence, and ocean observing.

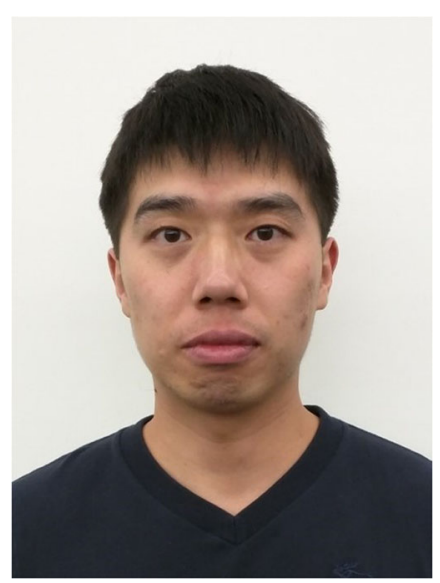

Li He received the B.E. and M.E. degrees from Huazhong University of Science and Technology, China, in 2006 and 2009 and the M.S. degree in electrical engineering from University of Nebraska - Lincoln, Lincoln, NE, USA, in 2011, respectively. $\mathrm{He}$ is currently an Engineer at Qualcomm Technologies, Inc., San Diego. His current research interests include computer vision, multimedia and machine learning.

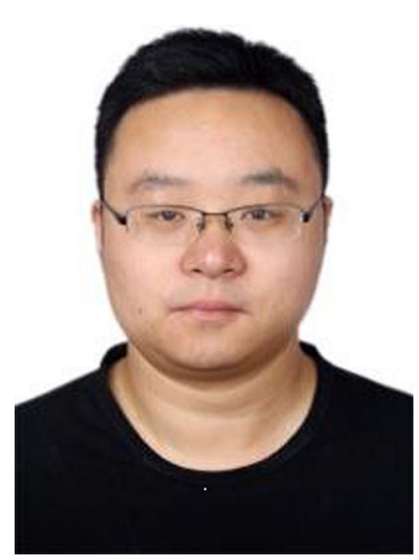

Quan Zhou received the M.S. degree and Ph.D. degree in communication and information system in 2006 and 2013, respectively, from Huazhong University of Science and Technology, China. $\mathrm{He}$ is now an associate professor of Nanjing University of Posts and Telecommunications. His research interests include computer vision and pattern recognition. He has published over 20 research papers in SCI journals (e.g., IEEE Transactions on Image Processing, IEEE Transactions on Multimedia, Pattern Recognition) and conference (ICIP, ICASSP, ACCV, and ICPR) in image processing and computer vision. He now serves as TPC member or chair of many international conferences and reviewer for a series of SCI journals, including IEEE Transactions on Image Processing, IEEE Transactions on Multimedia, IEEE Transactions on Circuits System for Video Technology, Pattern Recognition, and Neurocomputing. He is member of IEEE.

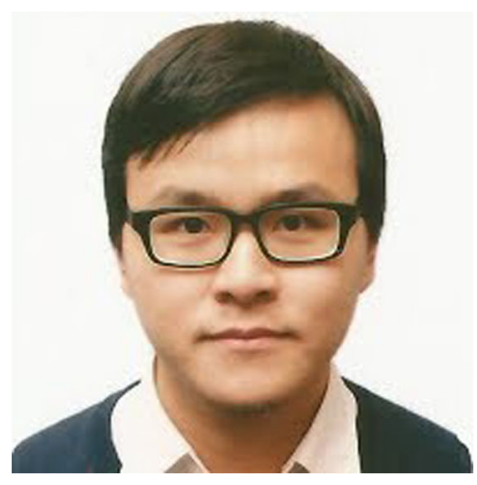

Zongyuan Ge received his Bachelor degree (with honours) in electrical engineering from the Australian National University, Australia in 2012. $\mathrm{He}$ is expecting to receive his doctor degree of engineering from the Queensland University of Technology, Australia, in 2016. He was a joint research student of Australian Centre for Robotic Vision and Queensland University of Technology. Currently, he is a research scientist at IBM Research, Australia. His research interests are in computer vision, face verification and finegrained image and video classification. His current research focuses on medical image analysis. 